\title{
METRIC PROPERTIES OF THE GROUP OF AREA PRESERVING DIFFEOMORPHISMS
}

\author{
MICHEL BENAIM AND JEAN-MARC GAMBAUDO
}

\begin{abstract}
Area preserving diffeomorphisms of the 2-disk which are identity near the boundary form a group $\mathcal{D}_{2}$ which can be equipped, using the $L^{2}$ norm on its Lie algebra, with a right invariant metric. With this metric the diameter of $\mathcal{D}_{2}$ is infinite. In this paper we show that $\mathcal{D}_{2}$ contains quasiisometric embeddings of any finitely generated free group and any finitely generated abelian free group.
\end{abstract}

\section{INTRODUCTION}

Let $\mathcal{D}_{2}$ be the group of smooth area preserving diffeomorphisms of the 2-disk which are identity near the boundary of the disk . A tangent vector to $\mathcal{D}_{2}$ at a point $\phi$ is a divergence free vector field $X_{\phi}$ (see [1] and [5]) whose $L^{2}$-norm is defined by

$$
\left\|X_{\phi}\right\|_{2}=\left(\int_{\mathbf{D}^{2}}\left\|X_{\phi}(x)\right\|^{2} d x\right)^{1 / 2}
$$

where $\|\cdot\|$ stands for the standard Euclidean norm. Consider a path $\left\{\phi_{t}\right\}_{t \in[0,1]}$ in $\mathcal{D}_{2}$ connecting two maps $\phi_{0}$ and $\phi_{1}$. The length of the path $\left\{\phi_{t}\right\}$ is given by the formula

$$
l_{2}\left(\left\{\phi_{t}\right\}\right)=\int_{0}^{1}\left\|\frac{d \phi_{t}}{d t}\right\|_{2} d t .
$$

Any two maps in $\mathcal{D}_{2}$ being connected by a path with finite length $l_{2}$, induces a distance on $\mathcal{D}_{2}$ defined by $d_{2}\left(\phi_{0}, \phi_{1}\right)=\inf l_{2}\left(\left\{\phi_{t}\right\}\right)$, where the infimum is taken over all paths joining $\phi_{0}$ to $\phi_{1}$. Notice that this distance is right invariant:

$$
d_{2}\left(\phi_{0} \circ \phi, \phi_{1} \circ \phi\right)=d_{2}\left(\phi_{0}, \phi_{1}\right) .
$$

Equipped with this distance, $\mathcal{D}_{2}$ has an infinite diameter (see [13], 6] and [1]). The group of area preserving diffeomorphisms is important in hydrodynamics; it is the configuration space of an incompressible 2-dimensional fluid (see [1]). The Euler-Lagrange equation associated to the metric $d_{2}$ corresponds to the equation of the motion of a perfect incompressible flow (Euler equation).

Finitely generated groups, i.e. groups generated with a finite number of generators obeying a finite number of relations, are another standard class of groups equipped with a right invariant metric. Let $\mathcal{G}$ be such a group, and $\mathcal{S}$ a system of generators of $\mathcal{G}$. The length $l_{\mathcal{S}}(g)$ of an element $g$ in $\mathcal{G}$ is the minimal number of

Received by the editors April 11, 2000 and, in revised form, October 30, 2000.

1991 Mathematics Subject Classification. Primary 20F36, 58B05, 58B25, 76A02.

Key words and phrases. Area preserving diffeomorphisms, braids, free groups, quasi-isometry. 
generators in $\mathcal{S}$ and their inverses needed to write $g$. It defines a right invariant natural metric on $\mathcal{G}$ :

$$
d_{\mathcal{S}}\left(g_{1}, g_{2}\right)=l_{\mathcal{S}}\left(g_{1} g_{2}^{-1}\right)
$$

The free group $\mathbf{F}_{n}$ and the abelian free group $\mathbf{Z}^{n}$ with $n$ generators are classical examples of finitely generated groups.

Let $\left(\mathcal{G}_{1}, d_{1}\right)$ and $\left(\mathcal{G}_{2}, d_{2}\right)$ be two metric groups, a morphism $m: \mathcal{G}_{1} \rightarrow \mathcal{G}_{2}$ is a quasi-expanding embedding if:

- $m$ is injective;

- there exists a pair of positive constants $k, k^{\prime}>0$ such that for any pair $g_{1}, g_{1}^{\prime}$ in $\mathcal{G}_{1}$ :

$$
d_{2}\left(m\left(g_{1}\right), m\left(g_{1}^{\prime}\right)\right) \geq k d_{1}\left(g_{1}, g_{1}^{\prime}\right)-k^{\prime} .
$$

An isomorphism $m$ between two metric groups is a quasi-isometry if $m$ and its inverse are quasi-expanding embeddings. A quasi-isometric embedding is a morphism which is a quasi-isometry onto its image. We refer the reader to [10] for more details and results concerning quasi-isometries.

In the case of a finitely generated group $\mathcal{G}$ equipped with 2 systems of generators $\mathcal{S}$ and $\mathcal{S}^{\prime}$, we have for any $g$ in $\mathcal{G}$ :

$$
\frac{1}{k\left(\mathcal{S}^{\prime}, \mathcal{S}\right)} l_{\mathcal{S}}(g) \leq l_{\mathcal{S}^{\prime}}(g) \leq k\left(\mathcal{S}, \mathcal{S}^{\prime}\right) l_{\mathcal{S}}(g)
$$

where $k\left(\mathcal{S}, \mathcal{S}^{\prime}\right)$ stands for the maximum over all the generators $s$ in $\mathcal{S}$ of the length $l_{\mathcal{S}^{\prime}}(s)$. Thus the identity map is a quasi-isometric isomorphism from $\left(\mathcal{G}, d_{\mathcal{S}}\right)$ to $\left(\mathcal{G}, d_{\mathcal{S}^{\prime}}\right)$ where $d_{\mathcal{S}}$ and $d_{\mathcal{S}^{\prime}}$ stand respectively for the right invariant metrics induced by $\mathcal{S}$ and $\mathcal{S}^{\prime}$. It follows that for a morphism of metric groups with values in (or taking values in) a finitely generated group $\left(\mathcal{G}, d_{\mathcal{S}}\right)$ (where $d_{S}$ is the right invariant metric induced by a system of generators $\mathcal{S}$ ) the property of being a quasi-isometric embedding does not depend on the set of generators.

This note is an attempt to understand the anatomy of the group $\mathcal{D}_{2}$ seen as a metric space. Since closed balls are not compact in $\mathcal{D}_{2}$, a direct approach using growth of balls seems pointless. Our point of view here is to show the complexity of $\mathcal{D}_{2}$ by showing that very different finitely generated groups can be embedded by quasi-isometric morphisms in $\mathcal{D}_{2}$. We prove the following result:

Theorem 1. Any finitely generated free group and any finitely generated abelian free group is quasi-isometrically embedded in $\mathcal{D}_{2}$.

The keys for this theorem rely on the following two facts that we will prove and make more explicit in the sequel:

- any finitely generated free group and any finitely generated abelian free group can be mapped by a quasi-isometric embedding in a braid group;

- the braid subgroups obtained this way can be in turn mapped by a quasiisometric embedding in the group $\mathcal{D}_{2}$.

Finally, we conclude with three remarks related to Theorem 10 and its possible generalizations.

\section{THE FREE GROUPS AND THE ABELIAN FREE GROUPS}

For any integer $n>0$ we denote by $\mathbf{F}_{n}\left(\right.$ resp. $\mathbf{Z}^{n}$ ) the free group (resp. the abelian free group) with $n$ generators $e_{1}, \ldots, e_{n}$ and $d_{n}$ the corresponding right 
invariant metric. For any pair of integers $2 \leq r \leq s$, there exists a quasi-isometric embedding from the (abelian) free group with $r$ generators to the (abelian) free group with $s$ generators. In the case of the free groups the converse is also true, more precisely we have the following standard result:

Lemma 1. For any $n \geq 2$, there exists a morphism from the free group with $n$ generators $\mathbf{F}_{n}$ to the free group with 2 generators $\mathbf{F}_{2}$ which is a quasi-isometric embedding.

Proof. For a given $n$, one can choose an integer $r$ large enough, and a system of $n$ elements in $\mathbf{F}_{2}, g_{1}, \ldots, g_{n}$, such that

- these words can be written using only the generators $e_{1}$ and $e_{2}$ of $\mathbf{F}_{2}$ (and not their inverses;

- they have a same length equal to $10 r$;

- for any pair $i, j$ in $1, \ldots, n$ the word $g_{i} g_{j}^{-1}$ has length at least $18 r$.

It follows that the morphism $\mu: \mathbf{F}_{n} \rightarrow \mathbf{F}_{2}$ which associates to each generator $e_{i}$ of $\mathbf{F}_{n}$ the element $g_{i}$ satisfies

$$
8 r d_{n}\left(f_{1}, f_{2}\right) \leq d_{2}\left(\mu\left(f_{1}\right), \mu\left(f_{2}\right)\right) \leq 10 r d_{n}\left(f_{1}, f_{2}\right),
$$

for any pair of elements $f_{1}, f_{2}$ in $\mathbf{F}_{n}$.

The second inequality shows that $\mu$ is an injective morphism; combined with the first one, we get that it is a quasi-isometric embedding.

\section{The BRAID GROUP AND ITS SUBgROUPS}

The Artin Braid group $\mathbf{B}_{n}$ is a group given by the set of generators $\sigma_{1}, \sigma_{2}, \ldots$, $\sigma_{n-1}$ and the relations

$$
\sigma_{i} \sigma_{j}=\sigma_{j} \sigma_{i}
$$

for all $i, j$ in $\{1, \ldots, n-1\}$ with $|i-j| \geq 2$ and

$$
\sigma_{i} \sigma_{i+1} \sigma_{i}=\sigma_{i+1} \sigma_{i} \sigma_{i+1}
$$

for all $i$ in $\{1, \ldots, n-2\}$. An element of this group is called $a$ braid.

A geometrical way to represent braids (see for instance [3] and [12]) consists in fixing the $2 n$ points $P_{i}=(i /(n-1)-1 / 2,0,1)$ and $Q_{i}=(i /(n-1)-1 / 2,0,0)$ for $i=0, \ldots, n-1$ in the solid cylinder $\mathbf{D}^{2} \times[0,1]$. A braid $\beta$ can be seen as the isotopy class of a system of $n$ non-intersecting arcs, joining each point $P_{i}$ to a point $Q_{\tau_{\beta}(i)}$, where $\tau_{\beta}$ is a permutation on $\{0, \ldots, n-1\}$, and such that the intersection of any of these arcs with any disk $\mathbf{D}^{2} \times\{t\}, t$ in $[0,1]$, consists in a unique point. With this representation, it is easy to understand what the group law, the generators and the relations mean.

There exists a classical surjective representation $\rho$ of the braid group $\mathbf{B}_{3}$ in the group $\mathbf{P S L}(2, \mathbf{Z})$ which is defined by

$$
\rho\left(\sigma_{1}\right)=s_{1} \text { and } \rho\left(\sigma_{2}\right)=s_{2},
$$

where $s_{1}$ and $s_{2}$ are respectively the representatives in $\mathbf{P S L}(2, \mathbf{Z})$ of the following two elements in $\mathbf{S L}(2, \mathbf{Z})$ :

$$
\left(\begin{array}{ll}
1 & 1 \\
0 & 1
\end{array}\right) \text { and }\left(\begin{array}{cc}
1 & 0 \\
-1 & 1
\end{array}\right)
$$


It is a representation since the only relation in $\mathbf{B}_{3}$

$$
\sigma_{1} \sigma_{2} \sigma_{1}=\sigma_{2} \sigma_{1} \sigma_{2}
$$

is preserved by $\rho$ :

$$
s_{1} s_{2} s_{1}=s_{2} s_{1} s_{2}
$$

It is surjective since $\mathbf{P S L}(2, \mathbf{Z})$ is generated by the two elements $s_{1}$ and $s_{2}$. The two elements in $\mathbf{P S L}(2, \mathbf{Z})$,

$$
a=s_{1} s_{2} \text { and } b=s_{1} s_{2} s_{1},
$$

satisfy $a^{3}=b^{2}=I d$ and generate $\operatorname{PSL}(2, \mathbf{Z})$ which actually is isomorphic to the free product of $\mathbf{Z} / 2 \mathbf{Z}$ and $\mathbf{Z} / 3 \mathbf{Z}$ :

$$
\operatorname{PSL}(2, \mathbf{Z})=\left\langle a, b ; a^{3}, b^{2}\right\rangle .
$$

The generators $s_{1}$ and $s_{2}$ can be written using $a$ and $b$ :

$$
s_{1}=a^{-1} b \text { and } s_{2}=b a^{-1} \text {. }
$$

In the particular case of the group $\mathbf{P S L}(2, \mathbf{Z})$ equipped with the two systems of generators $\left\{s_{1}, s_{2}\right\}$ and $\{a, b\}$, equation (1) reads

$$
\frac{1}{3} l_{\left\{s_{1}, s_{2}\right\}} \leq l_{\{a, b\}} \leq 2 l_{\left\{s_{1}, s_{2}\right\}} \text {. }
$$

The following proposition is a classical result. For the sake of completeness, we give it below followed by a short proof.

Proposition 1. The morphism $m$ from the free group $\mathbf{F}_{2}$ to $\mathbf{P S L}(2, \mathbf{Z})$ defined by

$$
m\left(e_{1}\right)=s_{1}^{2}=a^{-1} b a^{-1} b \text { and } m\left(e_{2}\right)=s_{2}^{2}=b a^{-1} b a^{-1},
$$

is a quasi-isometric embedding.

Proof. Consider a word $f$ in $\mathbf{F}_{2}$, written in a reduced form (i.e. with the smallest possible length) and let $f^{n}$ be the word in $\mathbf{F}_{2}$ with length $n<l_{\left\{e_{1}, e_{2}\right\}}(f)$ which coincides with $f$ truncated after its $n^{\text {th }}$ symbol. It is easy to check that

$$
l_{\{a, b\}}\left(m\left(f^{n+1}\right)\right) \geq l_{\{a, b\}}\left(m\left(f^{n}\right)\right)+1 .
$$

It follows that

(3) $l_{\{a, b\}}(m(f)) \geq l_{\left\{e_{1}, e_{2}\right\}}(f)-1+l_{\{a, b\}}\left(m\left(f^{1}\right)\right)=l_{\left\{e_{1}, e_{2}\right\}}(f)+3 \geq l_{\left\{e_{1}, e_{2}\right\}}(f)$.

On the other hand, for any element $f$ in $\mathbf{F}_{2}$ we have

$$
l_{\{a, b\}}(m(f)) \leq 4 l_{\left\{s_{1}^{2}, s_{2}^{2}\right\}}(m(f))=4 l_{\left\{e_{1}, e_{2}\right\}}(f) .
$$

This series of inequalities show that the morphism $m$ is a quasi-isometric embedding.

This quasi-isometric embedding can be easily visualized on the Caley graph of the group $\mathbf{P S L}(2, \mathbf{Z})$ (see this graph in [10]).

Corollary 1. The morphism $\bar{m}$ from the free group $\mathbf{F}_{2}$ to $\mathbf{B}_{3}$ defined by

$$
\bar{m}\left(e_{1}\right)=\sigma_{1}^{2} \text { and } \bar{m}\left(e_{2}\right)=\sigma_{2}^{2},
$$

is a quasi-isometric embedding. 
Proof. On one hand, we obviously have

$$
l_{\left\{\sigma_{1}, \sigma_{2}\right\}}(\bar{m}(f)) \leq 2 l_{\left\{e_{1}, e_{2}\right\}}(f) .
$$

On the other hand, we get from the relation

$$
m=\rho \circ \bar{m}
$$

that for any element $f$ in $\mathbf{F}_{2}$,

$$
l_{\left\{s_{1}, s_{2}\right\}}(m(f)) \leq l_{\left\{\sigma_{1}, \sigma_{2}\right\}}(\bar{m}(f)) .
$$

Combining Equations (2) and (3) we get

$$
\frac{1}{2} l_{\left\{e_{1}, e_{2}\right\}}(f) \leq \frac{1}{2} l_{\{a, b\}}(m(f)) \leq l_{\left\{s_{1}, s_{2}\right\}}(m(f)) .
$$

Thus,

$$
\frac{1}{2} l_{\left\{e_{1}, e_{2}\right\}}(f) \leq l_{\left\{\sigma_{1}, \sigma_{2}\right\}}(\bar{m}(f)) .
$$

These inequalities prove the corollary.

Remark 1. The embedding of the free group $\mathbf{F}_{2}$ in the braid group $\mathbf{B}_{3}$ maps $\mathbf{F}_{2}$ onto the subgroup of $\mathbf{B}_{3}$ which consists in all equivalence classes of systems of 3 arcs, the first one and the last one being vertical and the second one turning around the other two. This interpretation allows us to identify $\bar{m}\left(\mathbf{F}_{2}\right)$, hence $\mathbf{F}_{2}$, with the fundamental group of the 2-punctured disk.

From Lemma 1 and Corollary 1 we get

Corollary 2. Any finitely generated free group can be embedded in $\mathbf{B}_{3}$ by a quasiisometric embedding.

Consider now embeddings of the abelian free groups in the braid groups.

Proposition 2. For any $n \geq 1$, the morphism $\tilde{m}_{n}$ from the group $\mathbf{Z}^{n}$ to the braid group $\mathbf{B}_{2 n}$ defined for $i=1, \ldots, n$ by

$$
\tilde{m}_{n}\left(e_{i}\right)=\left(\sigma_{2 i-1}\right)^{2},
$$

is a quasi-isometric embedding 1 .1

Proof. Since two generators $\sigma_{i}$ and $\sigma_{j}$ of the braid group $\mathbf{B}_{n}$ commute when then are not consecutive $(|i-j| \neq 1)$, the morphism $\tilde{m}_{n}$ is well defined. Any element $z$ in $\tilde{m}_{n}\left(\mathbf{Z}^{n}\right)$ can be written in a unique way as the product:

$$
z=\sigma_{1}^{\alpha_{1}(z)} \sigma_{3}^{\alpha_{3}(z)} \sigma_{5}^{\alpha_{5}(z)} \ldots \sigma_{2 n-1}^{\alpha_{2 n-1}(z)}
$$

where the $\alpha_{j}(z)$ 's are even integers.

For $i=0, \ldots, n-1$, the map $z \rightarrow \alpha_{i}(z)$ is a morphism. By identifying $\mathbf{B}_{2}$ with $\mathbf{Z}$, this morphism corresponds to the forgetful map: $\tau_{i}: \mathbf{B}_{2 n} \rightarrow \mathbf{B}_{2}$ which consists in forgetting all the strands of a representative of an element in $\mathbf{B}_{n}$ but the $(2 i-1)^{t h}$ and $(2 i)^{t h}$ ones. This yields the equality:

$$
l_{\left\{\sigma_{1}, \ldots, \sigma_{2 n-1}\right\}}(z)=\sum_{i=0}^{i=n-1}\left|\alpha_{i}(z)\right| .
$$

\footnotetext{
${ }^{1}$ It is only for technical reasons that will be clear in the next section that we define $\tilde{m}_{n}\left(e_{i}\right)=$ $\left(\sigma_{2 i-1}\right)^{2}$ and not $\tilde{m}_{n}\left(e_{i}\right)=\left(\sigma_{2 i-1}\right)$.
} 
Thus for any $f$ in $\mathbf{Z}^{n}$,

$$
l_{\left\{\sigma_{1}, \ldots, \sigma_{2 n-1}\right\}}\left(\tilde{m}_{n}(f)\right)=2 l_{\left\{e_{1}, \ldots, e_{n}\right\}}(z) .
$$

\section{Area preserving Diffeomorphisms}

4.1. A lower bound for the distance between two maps. We recall in this paragraph some results that are developed in $[9$.

Let us fix again an element $\phi$ in $\mathcal{D}_{2}$ and an isotopy $\left\{\phi_{t}\right\}_{t \in[0,1]}$ in $\mathcal{D}_{2}$ connecting identity to $\phi$. To any pair of distinct points $x, y$ in $\mathbf{D}^{2}$ and to every $t \in[0,1]$, we associate the unit vector

$$
u(t, x, y)=\frac{\phi_{t}(y)-\phi_{t}(x)}{\left\|\phi_{t}(y)-\phi_{t}(x)\right\|}
$$

Lemma 2 ([9]). The integral

$$
\mathcal{G}\left(\left\{\phi_{t}\right\}\right)=\frac{1}{2 \pi} \int_{0}^{1} \int_{\mathbf{D}^{2} \times \mathbf{D}^{2}}\left\|\frac{d u}{d t}(t, x, y)\right\| d x d y d t
$$

is well defined.

Proof. An easy calculation yields

$$
\left\|\frac{d u}{d t}(t, x, y)\right\|=\frac{\left\|\left(\dot{\phi}_{t}(y)-\dot{\phi}_{t}(x)\right) \wedge\left(\phi_{t}(y)-\phi_{t}(x)\right)\right\|}{\left\|\phi_{t}(y)-\phi_{t}(x)\right\|^{2}},
$$

where $\wedge$ is the wedge product and $\dot{\phi}_{t}=\frac{\partial \phi_{t}}{\partial t}$. It follows that

$$
\left\|\frac{d u}{d t}(t, x, y)\right\| \leq \frac{2}{\left\|\phi_{t}(y)-\phi_{t}(x)\right\|} \sup _{(t, x) \in[0,1] \times \mathbf{D}^{2}}\left\|\dot{\phi}_{t}(x)\right\| .
$$

The quantity $\sup _{(t, x) \in[0,1] \times \mathbf{D}^{2}}\left\|\dot{\phi}_{t}(x)\right\|$ is bounded. Hence it is enough to show that since $\phi_{t}$ is area preserving for every $t$ in $[0,1]$,

$$
\int_{\mathbf{D}^{2} \times \mathbf{D}^{2}} \frac{d x d y}{\left\|\phi_{t}(y)-\phi_{t}(x)\right\|}=\int_{\mathbf{D}^{2} \times \mathbf{D}^{2}} \frac{d x d y}{\|y-x\|}
$$

and that this last integral converges.

We now introduce the quantity

$$
\mathcal{G}(\phi)=\inf \mathcal{G}\left(\left\{\phi_{t}\right\}\right)
$$

where the infimum is taken over all isotopies in $\mathcal{D}_{2}$ joining identity to $\phi$.

Lemma $3([9])$. There exists a constant $K>0$ such that for any map $\phi$ in $\mathcal{D}_{2}$

$$
\mathcal{G}(\phi) \leq K l_{2}(\phi)
$$

Proof. The quantity $\mathcal{G}\left(\left\{\phi_{t}\right\}\right)$ satisfies

$$
\mathcal{G}\left(\left\{\phi_{t}\right\}\right) \leq \frac{1}{\pi} \int_{0}^{1} \int_{\mathbf{D}^{2} \times \mathbf{D}^{2}} \frac{\left\|\dot{\phi}_{t}(x)\right\|}{\left\|\phi_{t}(y)-\phi_{t}(x)\right\|} d x d y d t .
$$

The Cauchy-Schwarz inequality gives

$$
G\left(\left\{\phi_{t}\right\}\right) \leq \frac{1}{\pi} \int_{0}^{1}\left\|\dot{\phi}_{t}\right\|_{2}\left\|I_{t}\right\|_{2} d t
$$


where

$$
I_{t}(x)=\int_{\mathbf{D}^{2}} \frac{d y}{\left\|\phi_{t}(y)-\phi_{t}(x)\right\|} .
$$

For every $t$ in $[0,1]$ the map $\phi_{t}$ is area preserving; consequently, the $L_{2}$-norm of $I_{t}$ satisfies

$$
\left\|I_{t}\right\|_{2}=\left(\int_{\mathbf{D}^{2}}\left(\int_{\mathbf{D}^{2}} \frac{d y}{\|x-y\|}\right)^{2} d x\right)^{1 / 2}<+\infty .
$$

Thus, for $K=\frac{1}{\pi}\left\|I_{t}\right\|_{2}$ we get

$$
\mathcal{G}\left(\left\{\phi_{t}\right\}\right) \leq K l_{2}\left(\left\{\phi_{t}\right\}\right) .
$$

It follows that

$$
\mathcal{G}(\phi) \leq K l_{2}(\phi)
$$

The quantity $\mathcal{G}(\phi)$ can be interpreted as follows. For an isotopy $\left\{\phi_{t}\right\}_{t \in[0,1]}$ connecting identity to $\phi$ and a pair of distinct points $x, y$ in $\mathbf{D}^{2}$, we consider the map

$$
\begin{aligned}
u_{x, y}:[0,1] & \rightarrow \mathbf{S}^{1} \\
t & \mapsto u(t, x, y) .
\end{aligned}
$$

The change of variables induced by the map $u_{x, y}$ leads to the equality

$$
\int_{0}^{1}\left\|\frac{d u_{x, y}}{d t}(t)\right\| d t=\int_{\mathbf{S}^{1}} \sharp\left\{u_{x, y}{ }^{-1}(\omega)\right\} d \omega,
$$

where $\sharp$ stands for cardinality. By integrating we get

$$
\mathcal{G}\left(\left\{\phi_{t}\right\}\right)=\frac{1}{2 \pi} \int_{\mathbf{D}^{2} \times \mathbf{D}^{2}} \int_{\mathbf{S}^{1}} \sharp\left\{u_{x, y}{ }^{-1}(\omega)\right\} d \omega d x d y .
$$

Consider the $2 \operatorname{arcs} \gamma_{x}: t \mapsto\left(\phi_{t}(x), t\right)$ and $\gamma_{y}: t \mapsto\left(\phi_{t}(y), t\right)$ in the cylinder $\mathbf{D}^{2} \times[0,1]$ and choose a direction $\omega$ in $\mathbf{S}^{1}$ tangent to $\mathbf{D}^{2}$. When projected in the direction of $\omega$ onto a plane orthogonal to $\omega, \gamma_{y}$ overcrosses $\sharp\left\{u_{x, y}{ }^{-1}(\omega)\right\}$ times $\gamma_{x}$. The integral

$$
C r_{\left\{\phi_{t}\right\}}(x, y)=\frac{1}{2 \pi} \int_{\mathbf{S}^{1}} \sharp\left\{u_{x, y}{ }^{-1}(\omega)\right\} d \omega
$$

is the averaged number of times the arc $\gamma_{y}$ overcrosses $\gamma_{x}$ over all directions $\omega$ tangent to $\mathbf{D}^{2} \cdot \mathcal{G}\left(\left\{\phi_{t}\right\}\right)$ is then the spatial average $\int_{\mathbf{D}^{2} \times \mathbf{D}^{2}} C r_{\left\{\phi_{t}\right\}}(x, y) d x d y$.

Changing the isotopy $\left\{\phi_{t}\right\}$ may reduce the averaged quantity of overcrossings $\mathcal{G}\left(\left\{\phi_{t}\right\}\right)$; the infimum is given by $\mathcal{G}(\phi)$.

4.2. A subgroup of $\mathcal{D}_{2}$. For $r$ small enough, consider, for $i=0, \ldots, n-1$, the $n$ disks $D_{i}$ in $\mathbf{D}^{2}$, with radius $r$ and centered at $P_{i}=(i /(n-1)-1 / 2,0)$. We define the subgroup $\mathcal{B}_{n}$ which consists in these maps whose restriction to the disks $D_{i}$ is identity for $i=0, \ldots, n-1$. There is a natural morphism $\mu_{n}$ from the group $\mathcal{B}_{n}$ to the braid group $\mathbf{B}_{n}$ which is defined as follows:

- choose a map $\phi$ in $\mathcal{B}_{n}$ and an isotopy $\left\{\phi_{t}\right\}_{t \in[0,1]}$ from identity to $\phi$; 
- a representative of the braid $\mu_{n}(\phi)$ consists in the system of $\operatorname{arcs}$ in $\mathbf{D}^{2} \times[0,1]$ :

$$
\bigcup_{i=0}^{i=n-1}\left\{\left(\phi_{t}\left(P_{i}\right), t\right), t \in[0,1]\right\}
$$

Lemma 4. Let $\phi$ be a map in $\mathcal{B}_{n}$. There exists a quantity $C(r) \geq 0$ which depends only on $r$ and goes to zero with $r$ such that

$$
l_{2}(\phi) \geq \frac{1}{K} l_{\left\{\sigma_{1}, \ldots, \sigma_{n-1}\right\}}\left(\mu_{n}(\phi)\right)(1-C(r)) \times\left(\operatorname{area} D_{0}\right)^{2} .
$$

Proof. Let $\left\{\phi_{t}\right\}_{t \in[0,1]}$ in $\mathcal{D}_{2}$ be an isotopy connecting identity to $\phi$. The integral $\mathcal{G}\left(\left\{\phi_{t}\right\}\right)$ reads

$$
\mathcal{G}\left(\left\{\phi_{t}\right\}\right)=\int_{\mathbf{D}^{2} \times \mathbf{D}^{2}} \operatorname{Cr}_{\left\{\phi_{t}\right\}}(x, y) d x d y
$$

and consequently

$$
\mathcal{G}\left(\left\{\phi_{t}\right\}\right) \geq \sum_{i \neq j} \int_{D_{i} \times D_{j}} C r_{\left\{\phi_{t}\right\}}(x, y) d x d y=\int_{D_{0} \times D_{0}}\left(\sum_{i \neq j} C r_{\left\{\phi_{t}\right\}}\left(T_{i}(x), T_{j}(y)\right)\right) d x d y
$$

where $T_{i}$ stands for the translation which maps $D_{0}$ onto $D_{i}$.

For $(x, y, \omega) \in D_{0} \times D_{0} \times \mathbf{S}^{1}$, let $\mathcal{U}_{\phi}(x, y, \omega)$ be the minimum over all isotopies in $\mathcal{D}_{2}$ connecting identity to $\phi$ of the quantity

$$
\sum_{i \neq j} \sharp\left\{u_{T_{i}(x), T_{j}(y)}^{-1}(\omega)\right\},
$$

We have

$$
\mathcal{G}(\phi) \geq \frac{1}{2 \pi} \int_{\mathcal{S}_{1}} \int_{D_{0} \times D_{0}} \mathcal{U}_{\phi}(x, y, \omega) d \omega d x d y .
$$

Let $M(r)$ be the set of $\omega$ 's for which the orthogonal projections (in the direction $\omega)$ of the disks $D_{i}$ overlap. We denote by $2 \pi C(r)$ the measure of the set $M(r)$. One can easily check that $C(r)$ goes to zero with $r$. It is also clear that for $\omega$ in $\mathcal{S}^{1} \backslash M(r)$

$$
\mathcal{U}_{\phi}(x, y, \omega) \geq l_{\left\{\sigma_{1}, \ldots, \sigma_{n-1}\right\}}\left(\mu_{n}(\phi)\right) .
$$

By integrating over $\mathcal{S}^{1} \backslash M(r)$ we get

$$
\mathcal{G}(\phi) \geq l_{\left\{\sigma_{1}, \ldots, \sigma_{n-1}\right\}}\left(\mu_{n}(\phi)\right)(1-C(r)) \times\left(\operatorname{area} D_{0}\right)^{2} .
$$

\section{Proof of Theorem 1}

Consider in $\mathcal{B}_{3}$ two maps $\Theta_{1}$ and $\Theta_{2}$ such that

$$
\mu_{3}\left(\Theta_{1}\right)=\sigma_{1}^{2} \text { and } \mu_{3}\left(\Theta_{2}\right)=\sigma_{2}^{2} \text {. }
$$

The morphism $\pi: \mathbf{F}_{2} \rightarrow \mathcal{D}_{2}$ defined by

$$
\pi\left(e_{1}\right)=\Theta_{1} \text { and } \pi\left(e_{2}\right)=\Theta_{2},
$$

is injective since $\mu_{3} \circ \pi=\bar{m}$ is injective. For any $f$ in $\mathbf{F}_{2}$, it also satisfies

$$
\begin{aligned}
l_{2}(\pi(f)) & \geq \frac{1}{K} l_{\left\{\sigma_{1}, \sigma_{2}\right\}} \mu_{3}(\pi(f))(1-C(r)) \times\left(\text { area } D_{0}\right)^{2} \\
& \geq \frac{1}{2 K} l_{\left\{e_{1}, e_{2}\right\}}(f)(1-C(r)) \times\left(\text { area } D_{0}\right)^{2}
\end{aligned}
$$


the left inequality coming from Lemma 4, the right one from the second inequality in the proof of Corollary 1 .

On the other hand, for

$$
f=e_{\epsilon_{1}}^{\alpha_{1}} e_{\epsilon_{2}}^{\alpha_{2}} \ldots e_{\epsilon_{n}}^{\alpha_{n}}
$$

where $\epsilon_{i}=1$ or 2 , we have

$$
l_{2}(\pi(f)) \leq \sum_{i=1}^{n}\left|\alpha_{i}\right| l_{2}\left(\pi\left(e_{\epsilon_{i}}\right)\right) \leq l_{e_{1}, e_{2}}(f) \times \max _{i \in\{1,2\}} l_{2}\left(\pi\left(e_{i}\right)\right) .
$$

Using Lemma 1 we also get a quasi-isometric embedding of $\mathbf{F}_{n}$ in $\mathcal{D}_{2}$ for any $n \geq 2$. This proves Theorem 1 for finitely generated free groups.

Consider now $n$ maps $\Omega_{1}, \ldots, \Omega_{n}$ in $\mathcal{B}_{2 n}$ such that

- $\mu_{2 n}\left(\Omega_{i}\right)=\sigma_{2 i-1}^{2}$ for $i=1, \ldots, n$;

- the maps $\Omega_{i}$ are different from identity on disjoint supports, thus they commute.

Since the maps $\Omega_{i}$ commute, the morphism $\tilde{\pi}: \mathbf{Z}^{n} \rightarrow \mathcal{D}_{2}$ defined for $i=1, \ldots, n$, by

$$
\tilde{\pi}\left(e_{i}\right)=\Omega_{i}
$$

is well defined. It is injective since $\mu_{2 n} \circ \tilde{\pi}=\tilde{m}_{n}$ is injective. For any $f$ in $\mathbf{Z}^{n}$, it also satisfies

$$
\begin{aligned}
l_{2}(\tilde{\pi}(f)) & \geq \frac{1}{K} l_{\left\{\sigma_{1}, \ldots, \sigma_{2 n-1}\right\}} \mu_{2 n}(\tilde{\pi}(f))(1-C(r)) \times\left(\text { area } D_{0}\right)^{2} \\
& =\frac{2}{K} l_{\left\{e_{1}, \ldots, e_{n}\right\}}(f)(1-C(r)) \times\left(\operatorname{area} D_{0}\right)^{2} ;
\end{aligned}
$$

the left inequality coming from Lemma 4 the right one from the last inequality in the proof of Proposition 2

On the other hand, given

$$
f=e_{1}^{\alpha_{1}} e_{2}^{\alpha_{2}} \ldots e_{n}^{\alpha_{n}}
$$

we have

$$
l_{2}(\tilde{\pi}(f)) \leq \sum_{i=1}^{n}\left|\alpha_{i}\right| l_{2}\left(\tilde{\pi}\left(e_{i}\right)\right) \leq l_{e_{1}, \ldots, e_{n}}(f) \times \max _{i \in\{1, \ldots, n\}} l_{2}\left(\tilde{\pi}\left(e_{i}\right)\right) .
$$

Thus for any $n \geq 1$, we get a quasi-isometric embedding of $\mathbf{Z}^{n}$ in $\mathcal{D}_{2}$. This achieves the proof of Theorem 1.

\section{FinAL REMARKS}

6.1. Random walks on $\mathcal{D}_{2}$. Let $G$ be a group equipped with a right invariant metric $d$. Consider the Borel $\sigma$-field of $G, \mathcal{B}(G)$, and let $\mu: \mathcal{B}(G) \mapsto[0,1]$ be a Borel probability measure on $G$. We can associate to $\mu$ the random walk on $\mathcal{D}_{2}$ $X=\left\{X^{n}\right\}_{n \in \mathbf{Z}^{+}}$defined by

$$
\begin{gathered}
X^{0}=I d, \\
X^{n}=g_{n} \cdot g_{n-1} \ldots g_{1} \quad \text { for } n \geq 1,
\end{gathered}
$$

where $\left\{g_{i}\right\}_{i \in \mathbf{Z}^{+}}$is a sequence of independent random variables defined on some probability space $(\Omega, \mathcal{F}, P)$ and identically distributed according to $\mu$. 
For any random variable $X: \Omega \mapsto \mathbf{R}$ and $p \geq 1$ we let $\|X\|_{p}=\left(\int_{\Omega}|X|^{p} d P\right)^{1 / p}$. $L^{p}(P)$ is the set of random variables $X$ for which $\|X\|_{p}<\infty$. For $X \in L^{1}(P)$ we let $E(X)=\int_{\Omega} X d P$ denote the mathematical expectation of $X$.

Proposition 6.1. Suppose $E\left(d\left(X^{1}, I d\right)\right)=\int_{\mathbf{R}} d(g, I d) \mu(d g)<\infty$, and let

$$
\gamma_{1}=\inf _{n \in \mathbf{Z}^{+}} \frac{1}{n} E\left(d\left(X^{n}, I d\right)\right) .
$$

Then

$$
\lim _{n \rightarrow \infty} \frac{l_{2}\left(d\left(X^{n}, I d\right)\right)}{n}=\gamma_{1}
$$

P-almost surely.

Proof. The proof is standard. We can always suppose that $(\Omega, \mathcal{F}, P)$ is the canonical space where $\Omega=G^{\mathbf{Z}^{+}}, \mathcal{F}=\mathcal{B}\left(G^{\mathbf{Z}^{+}}\right)$and $P=\mu^{\otimes \mathbf{Z}^{+}}$is the product measure. Let $\Theta: \Omega \mapsto \Omega$ denote the shift defined by $\Theta(\mathbf{g})_{i}=g_{i+1}$ where $\mathbf{g}=\left\{g_{i}\right\}_{i \in \mathbf{Z}^{+}}$and let $\tau_{n}(\mathbf{g})=d\left(g_{n} . g_{n-1} \ldots g_{1}, I d\right)$. The right invariance of $d$ implies that

$$
\begin{aligned}
\tau_{n+m} & (\mathbf{g})=d\left(g_{n+m} \ldots g_{1}, I d\right) \\
& =d\left(g_{n+m} \ldots g_{n+1},\left(g_{n} \ldots g_{1}\right)^{-1}\right) \\
\leq & \tau_{n}(\mathbf{g})+\tau_{m}\left(\Theta^{n}(\mathbf{g})\right)
\end{aligned}
$$

Hence Proposition 6.1 follows from Kingman's subadditive Ergodic Theorem [1].

The quantity $\gamma_{1}$ is called the linear escape rate of the random walk.

Examples. $\quad$ Consider the free group $\mathbf{F}_{d}$ with generators $\mathcal{S}=\left\{e_{1}, \ldots, e_{d}\right\}$ and equipped with the right invariant metric induced by the word length. Charge the generators and their inverse with an equidistributed measure $\left(\mu\left(e_{i}\right)=\right.$ $\left.\mu\left(e_{i}^{-1}\right)=\frac{1}{d}\right)$ and consider the random walk on this group which consists in starting form an element chosen at random in $\mathcal{S} \cup \mathcal{S}^{-1}$ and mutiplying at each step on the left by an element chosen at random in $\mathcal{S} \cup \mathcal{S}^{-1}$. It is easy to check that the linear rate of escape in this particular case satifies

$$
\gamma_{1}=1-\frac{1}{d}
$$

- For a similar random walk on the abelian free group $\mathbf{Z}^{d}$ it is also known that the linear escape rate vanishes $\left(\gamma_{1}=0\right)$.

- From Theorem 11 we get that for any positive integer $d$, there exist $d$ maps $\Theta_{1}, \ldots, \Theta_{d}$ in $\mathcal{D}_{2}$ such that the group generated by $\Theta_{1}, \ldots, \Theta_{d}$ is quasiisometric to the free group. It implies that the associated random walk on this group has a strictly positive linear escape rate.

- From Theorem 11 we also get that for any positive integer $d$, there exist $d$ maps $\Omega_{1}, \ldots, \Omega_{d}$ in $\mathcal{D}_{2}$ such that the group generated by $\Omega_{1}, \ldots, \Omega_{d}$ is quasiisometric to the abelian free group $\mathbf{Z}^{d}$. It implies that the associated random walk on this group has a linear escape rate which vanishes.

Question 1. For any $d>1$ and for any generic choice of maps $\phi_{1}, \ldots, \phi_{d}$ in $\mathcal{D}_{2}$, consider the subgroup $\mathcal{D}_{2}\left(\phi_{1}, \ldots, \phi_{d}\right)$ of $\mathcal{D}_{2}$ generated by $\left\{\phi_{1}, \ldots, \phi_{d}\right\}$. Is this subgroup a quasi-isometric embedding of the free group $\mathbf{F}_{d}$ ? More generally, does the random walk which gives an equal probability to each generator and their inverse, have a non-zero linear rate of escape? 
6.2. Embeddings in the kernel of the Calabi morphism. Consider a map $\phi$ in $\mathcal{D}_{2}$ and a 1 -form $\alpha$ on $\mathbf{D}^{2}$ which is a primitive of the area 2 -form. Since $\phi$ is area preserving, the form $\phi^{\star} \alpha-\alpha$ is closed and vanishes near the boundary of $\mathbf{D}^{2}$. Thus, there exists a unique function $H(\phi, \alpha): \mathbf{D}^{2} \rightarrow \mathbf{R}$, which vanishes near the boundary of $\mathbf{D}^{2}$ and such that

$$
d H(\phi, \alpha)=\phi^{\star} \alpha-\alpha .
$$

The Calabi invariant [4] of $\phi$ is defined by

$$
\mathcal{C}(\phi)=\int_{\mathbf{D}^{2}} H(\phi, \alpha)
$$

It is easy to verify that this integral does not depend on the choice of the primitive $\alpha$ and that the Calabi invariant is actually a morphism from $\mathcal{D}_{2}$ to $\mathbf{R}$ :

$$
\mathcal{C}\left(\phi_{0} \circ \phi_{1}\right)=\mathcal{C}\left(\phi_{0}\right)+\mathcal{C}\left(\phi_{1}\right)
$$

A. Banyaga 2] proved that the kernel of this morphism, $\operatorname{ker} \mathcal{C}$, is a simple group and Y. Eliashberg and T. Ratiu [6] showed that its subgroup has an infinite diameter (see also [9]).

There is a second definition of the Calabi invariant which is due to A. Fathi [7] (see also [8]) and which can be seen as an estimate in terms of braiding of pairs of orbits. Consider an isotopy $\left\{\phi_{t}\right\}_{t \in[0,1]}$ in $\mathcal{D}_{2}$ connecting identity to $\phi$. The map

$$
A n g_{\phi}: \mathbf{D}^{2} \times \mathbf{D}^{2} \backslash \Delta \rightarrow \mathbf{R},
$$

(where $\Delta$ stands for the diagonal) which associates to any pair of points $x \neq y$ in $\mathbf{D}^{2}$ the angular variation of the vector $\overrightarrow{\phi_{t}(x) \phi_{t}(y)}$ when $t$ goes from 0 to 1 , does not depend on the choice of the isotopy and is bounded where it is defined. The Calabi invariant is the integral of this function, i.e.:

$$
\mathcal{C}(\phi)=\int_{\mathbf{D}^{2} \times \mathbf{D}^{2}} \operatorname{Ang}_{\phi}(x, y) d x d y .
$$

With this second definition, $\operatorname{Ker} \mathcal{C}$ is the subgroup of area preserving maps whose angular variation of a pair of points vanishes in average. Using this second geometric interpretation, Theorem 1 can be made more precise:

Theorem 2. Any finitely generated free group and any finitely generated abelian free group is quasi-isometrically embedded in $\operatorname{KerC}$.

We only give here the main lines of the proof and insist on its differences with the proof of Theorem 1 .

Consider the morphism $m^{\prime}: \mathbf{F}_{2} \rightarrow \mathbf{B}_{5}$ which maps the generator $e_{1}$ on $\sigma_{1}^{2} \sigma_{3}^{-2}$ and $e_{2}$ on $\sigma_{2}^{2} \sigma_{4}^{-2}$. Using the same technics as the ones we used in the proof of Theorem 1 we get that $m^{\prime}$ is a quasi-isometric embedding. It is possible to find two maps in $\mathcal{B}_{5}, \Theta_{1}^{\prime}$ and $\Theta_{2}^{\prime}$ such that

- $\mu_{6}\left(\Theta_{1}^{\prime}\right)=\sigma_{1}^{2} \sigma_{3}^{-2}$ and $\mu_{6}\left(\Theta_{2}^{\prime}\right)=\sigma_{2}^{2} \sigma_{4}^{-2}$;

- $\Theta_{1}^{\prime}$ and $\Theta_{2}^{\prime}$ are in $\operatorname{Ker} \mathcal{C}$.

Similarly to the proof of Theorem 1, we prove that the morphism $\pi^{\prime}: \mathbf{F}_{2} \rightarrow \operatorname{Ker} \mathcal{C}$ defined by $\pi^{\prime}\left(e_{1}\right)=\Theta_{1}^{\prime}$ and $\pi^{\prime}\left(e_{2}\right)=\Theta_{2}^{\prime}$ is a quasi-isometric embedding. Using Corollary [, we get quasi-isometric embeddings of any finitely generated free group in $\operatorname{KerC}$. A similar construction works for finitely generated abelian free groups. 
6.3. Symplectomorphisms in higher dimensions. Let $n>0$ and consider the group $\mathcal{D}_{2 n}$ of diffeomorphisms of the unit ball in the $2 n$-dimensional space $B^{2 n}$ which preserve the standard symplectic 2-form $\omega=\sum_{i=1}^{n} d x_{i} \wedge d y_{i}$, and are identity near the boundary $\partial B^{2 n}$. This group, like $\mathcal{D}_{2}$, can be equipped with a right invariant metric. With this metric, $\mathcal{D}_{2 n}$ also has an infinite diameter [6].

Question 2. Does a quasi-isometric embedding of any finitely generated free group and any finitely generated abelian free group in $\mathcal{D}_{2 n}$ exist for all $n>0$ ?

\section{Acknowledgments}

It is a pleasure to thank J. González-Meneses and L. Paris for helpful discussions on braid groups.

\section{REFERENCES}

[1] Arnold, V. And Khesin, B. Topological methods in hydrodynamics. Applied Mathematical Sciences, 125, (1998). MR 99b:58002

[2] Banyaga, A.: On the group of diffeomorphisms preserving an exact symplectic form, in Differential topology, Varenna (1976), 5-9. MR 83g:58006

[3] Birman, J. Braids, Links and Mapping Class Groups. Annals of Math. Studies 82, Princeton University Press, (1974) Erratum, 1975. MR 51:11477; MR 54:13894

[4] Calabi, E.: On the group of automorphisms of a symplectic manifold, in Problems in analysis, Symposium in honor of S. Bochner, R. C. Gunning, Ed., Princeton Univ. Press, Princeton (1970), 1-26. MR 50:3268

[5] Ebin, D. G. and Marsden, J.: Groups of diffeomorphisms and the notion of an incompressible fluid, Ann. of Math. 92, (1970), 102-163.

[6] Eliashberg, Y. and Ratiu, T.: The diameter of the symplectomorphism group is infinite, Invent. Math. 103, (1991), 327-340. MR 92a:58018

[7] FAthi, A.: Transformations et homéomorphismes préservant la mesure. Systèmes dynamiques minimaux., Thèse Orsay (1980).

[8] Gambaudo, J.-M. and Ghys, É.: Enlacements asymptotiques, Topology 36, (1997), 13551379. MR 98f:57050

[9] Gambaudo, J.-M. and Lagrange, M.: Topological lower bounds on the distance between area preserving diffeomorphisms, Bol. Soc. Brasil. Mat. 31, (2000), 1-19. CMP 2000:12

[10] Ghys, É. ANd DE LA HARPe, P. Sur les groupes hyperboliques d'après Mikhael Gromov, Ghys, É and de la Harpe, eds., Progress in Mathematics 83, Birkhauser, (1990). MR 92f:53050

[11] Kingman, J. F. C.: The ergodic theory of subadditive stochastic processes, J. Royal Stat. Soc., 30, (1968), 499-510. MR 40:8114

[12] Murasugi, K. Knot theory and its applications. Translated from the 1993 Japanese original by Bohdan Kurpita. Birkhäuser Boston, Inc., Boston, (1996). MR 97g:57011

[13] Shnirelman, A.: The geometry of the group of diffeomorphisms and the dynamics of an ideal incompressible fluid, Matem. Sbornik 128, (1985), 82-109; English transl: Math. USSR, Sbornik 56 (1987), 79-105. MR 87d:58034

Université de Cergy Pontoise, Laboratoire de Mathématiques, 2, avenue Adolphe Chauvin, 95302 Cergy-Pontoise Cedex, France

E-mail address: benaim@math.u-cergy.fr

Université de Bourgogne, Laboratoire de Topologie, UMR CNRS 5584, B.P. 4787021078-Dijon CEDEX, France 